\title{
Bubble entrapment during the recoil of an impacting droplet
}

\author{
Thanh-Vinh Nguyen ${ }^{1}$ and Masaaki Ichiki ${ }^{1}$
}

\begin{abstract}
When a droplet impacts a (super-)hydrophobic surface, there is a range of Weber numbers within which bubble entrapment will occur during droplet recoil due to closure of the air cavity developed when the droplet spreads out during the impact. In this study, we studied bubble entrapment using a microelectromechanical system (MEMS)-based acoustic sensor fabricated on a substrate. We found that bubble entrapment is followed by an acoustic vibration that can be detected by the sensor. Moreover, the frequency of the vibration is inversely proportional to the radius of the droplet, which indicates that this vibration is the resonant oscillation of the bubble. Therefore, the MEMS-based acoustic sensor can be used not only to detect but also to measure the size of the entrapped bubble. Finally, we demonstrated that it is possible to prevent bubble formation by allowing the air to escape to the underside of the droplet contact area. This can be done by creating through-holes on the substrate or decorating the substrate with sufficiently large textures.
\end{abstract}

\section{Introduction}

Droplet impact on a rigid substrate has been the topic of various studies because of its fascinating dynamics as well as its important role in a wide range of practical applications $^{1,2}$. When a droplet impacts a solid substrate, bubble entrapment can occur at the beginning of the impact or during the liquid recoil after the spread of the droplet on the substrate. It was shown that prior to the contact of the liquid with the substrate, the droplet slides on a very thin air film sandwiched between the droplet and the substrate ${ }^{3-9}$, and then the liquid/solid contact causes the air film to be trapped, resulting in the formation of a bubble inside the droplet ${ }^{3-5,10-12}$. However, this process is not the only mechanism of impactinduced bubble entrapment. In fact, within a specific range of Weber numbers (defined as $\mathrm{We}=\left(\rho D V^{2}\right) / \sigma$, where $\rho, \sigma, D$, and $V$ are the liquid density, liquid surface tension, droplet diameter, and impact velocity, respectively), the capillary wave occurring on the droplet surface

Correspondence: Thanh-Vinh Nguyen (vinh.nguyen@aist.go.jp)

'Sensing System Research Center, National Institute of Advanced Industrial Science and Technology (AIST), Japan, 1-2-1 Namiki, Tsukuba-shi, Ibaraki 3058564, Japan can cause the contact area center to dry out ${ }^{13}$; the recoil liquid then causes the closure of the air cavity, which eventually also leads to bubble entrapment ${ }^{14-18}$, as illustrated in Fig. 1a. This type of bubble entrapment during the recoiling stage of the droplet impact occurs when the contact angle between the liquid and the substrate is high (100 degrees or higher $)^{15}$.

In general, bubble entrapment is undesirable in many applications in which droplet impact is essential, such as in inkjet printing and spray cooling. However, the dropletentrapped air bubble can also be advantageous in some particular applications; for example, oscillation of the bubble entrapped inside a droplet can be used to enhance droplet mixing ${ }^{19}$. In that sense, droplet impact can be used as an effective method for rapidly creating a bubble inside the droplet. To achieve the best efficiency of bubble oscillation-based droplet mixing, it is necessary to know the size of the bubble to determine the frequency of the external acoustic vibration.

Here, we propose a method using a MEMS-based acoustic sensor for detecting bubble entrapment and simultaneously measuring the bubble size, as shown in Fig. 1b. The bubble entrapment during the recoil phase of 

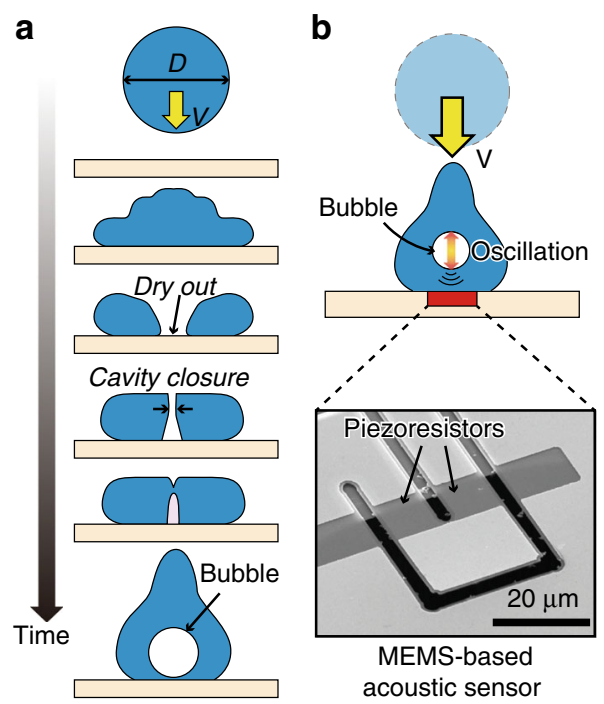

Fig. 1 Proposed method using MEMS-based acoustic sensor to study bubble entrapment during the recoil of droplet impact. a Mechanism of bubble entrapment during the recoil of droplet impact on a solid substrate. $\mathbf{b}$ Proposed method to study bubble entrapment

the droplet impact is a result of air cavity closure followed by the formation of a singularity jet, which causes the bubble to oscillate at its natural frequency determined by the size of the bubble. Therefore, by directly measuring the bubble oscillation using the MEMS-based acoustic sensor, it is possible to gain quantitative information on the bubble size. On the other hand, because the bubble is formed as the air cavity is sealed, we investigate the possibility of preventing bubble entrapment by letting the air escape, for example, to the underside of the contact area by using substrates that contain through-holes or that are decorated with sufficiently large textures.

\section{Results and discussion}

\section{Measurement of bubble entrapment}

Using the fabricated sensor chip, we measured the impacting force and acoustic signal during the impact of water droplets on the sensor chip at different velocities. In each measurement, the location of the droplet was adjusted such that the cantilever was located at the center of the contact area. Figure 2a and (b) shows the responses of the cantilever and snapshots of the high-speed camera in the cases, without bubble entrapment (droplet diameter: $2.2 \mathrm{~mm}$; impact velocity: $0.64 \mathrm{~m} / \mathrm{s}$ ) and with bubble entrapment (droplet diameter: $2.2 \mathrm{~mm}$, impact velocity: $0.50 \mathrm{~m} / \mathrm{s}$ ). The corresponding Supplementary videos S1 and S2 are provided in the Supplementary Information. The occurrence of bubble entrapment can be clearly observed in Supplementary video S2, but not in Supplementary video S1. For both cases, the cantilever's resistance changed immediately after the droplet hit the sensor
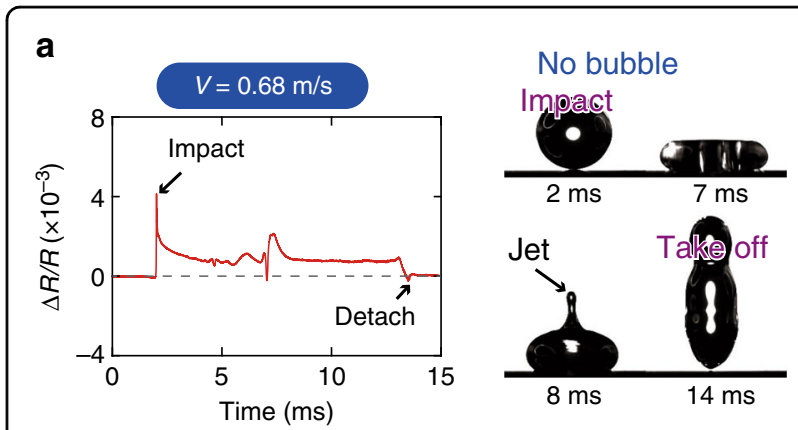

b
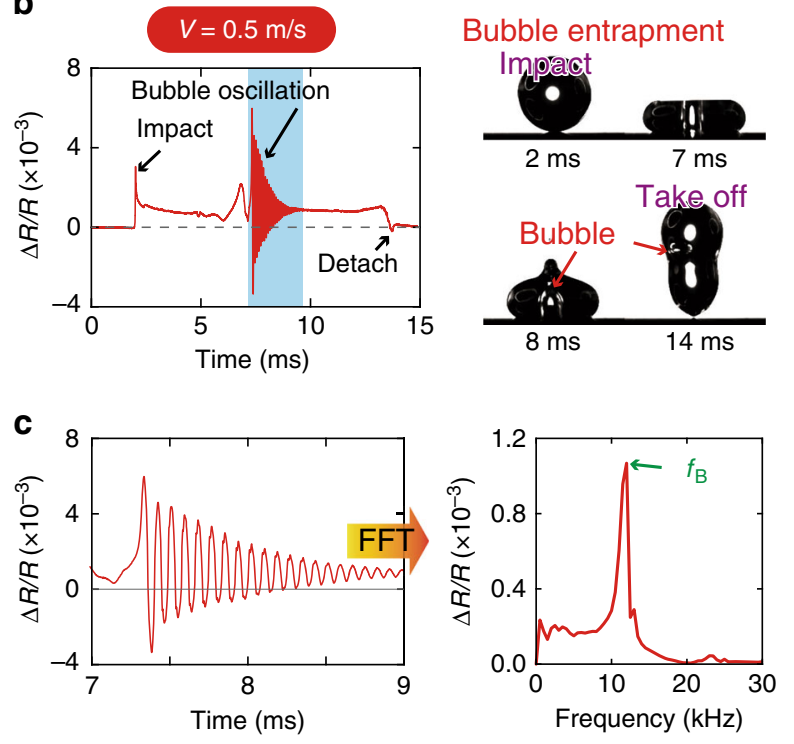

Fig. 2 Sensor outputs during the impacts of droplets on the sensor substrate. Responses of the MEMS-based acoustic sensor and images of the high-speed camera (a) in the case of no bubble entrapment and (b) in the case with bubble entrapment. c Acoustic vibration recorded by the sensor signal and its frequency characteristics

chip owing to the pressure caused by the impact. This resistance change was larger for higher impact velocities, as the pressure increases with increasing impact velocity. The most important difference between the signals of the cantilever in the two cases is the vibration presented in the sensor signal when bubble entrapment occurred. A zoomed-in view of the vibration and its frequency characteristics is shown in Fig. 2c (see also Supplementary video S3). From the frequency characteristics, the frequency of the vibration can be obtained as $f_{\mathrm{B}}$, which was $\sim 12 \mathrm{kHz}$ for the signal shown in Fig. 2c.

\section{Relationship between the bubble size and the frequency of} the acoustic signal detected by the sensor

The measurement was repeated for different droplet sizes and impact velocities to characterize different sizes of entrapped bubbles. For each entrapped bubble, its 


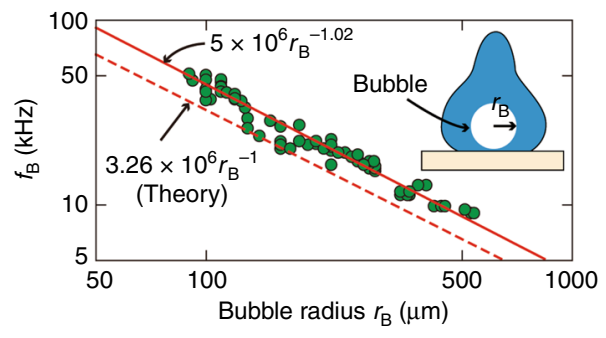

Fig. 3 Bubble size vs. frequency of the sensor signal. Relationship between the frequency of the vibration measured by the sensor and the radius of the bubble

radius $R_{\mathrm{B}}$ was calculated from the images of the highspeed camera. Figure 3 shows the relationship between the radius $R_{\mathrm{B}}$ of the bubble and the frequency $f_{\mathrm{B}}$ of the vibration. The solid line in the graph represents the fitting of the data with the function $y=a x^{b}$, where $a$ and $b$ are the fitting parameters. The result indicates that the frequency of vibration is inversely proportional to the bubble radius, which agrees with the relationship between the natural resonant frequency and the size of the bubble. In fact, neglecting the effect of surface tension and viscosity is possible because the viscosity of water is small and the sizes of the bubbles in our experiment were relatively large (diameter $>100 \mu \mathrm{m}$ ), so that the surface-tensioninduced component of the bubble inner pressure becomes sufficiently small in comparison with the ambient pressure. The theoretical relationship between the radius of an ideally spherical bubble and its natural resonant frequency is $^{20-22}$ :

$$
f_{\mathrm{B}}=\frac{1}{2 \pi R_{\mathrm{B}}} \sqrt{\frac{3 \gamma P}{\rho}}
$$

where $R_{\mathrm{B}}, \gamma, \rho$, and $P$ represent the bubble radius, polytropic coefficient, density of the liquid, and the ambient pressure, respectively. Using $\gamma=1.4, \rho=$ $1000 \mathrm{~kg} / \mathrm{m}^{3}$, and $P=10^{5} \mathrm{~Pa}$, we obtain

$$
f_{\mathrm{B}}=\sim \frac{3.26 \times 10^{6}}{R_{\mathrm{B}}}
$$

where the unit of $R_{\mathrm{B}}$ is $\mu \mathrm{m}$. Eq. (2) is represented by the dashed line in the graph of Fig. 3.

The agreement in the scaling law between the measured data and Eq. (2) indicates that the vibration measured by the sensor is indeed the natural frequency of the bubble. The difference in the coefficient parameters of $5 \times 10^{6}$ (measured) and $3.3 \times 10^{6}$ (theoretical) may be due to the error when measuring the radius of the bubbles entrapped inside the droplets from the images; that is, the droplet acts as an optical lens owing to its natural spherical shape, and the bubbles in the captured images appear to be larger than they actually are. Nevertheless, it is important to note that in the bubble application to enhance droplet mixing, the information by the sensor on the natural frequency of the bubble is what is required to determine the frequency of the external acoustic actuation. In other words, the proposed method can be used to rapidly entrap a bubble inside the droplet and then determine the optimized frequency of the acoustic actuation needed to achieve the most efficient mixing.

\section{Effect of the substrate morphology}

We investigate the effect of the substrate morphology on the occurrence of bubble entrapment using MEMS sensors whose surfaces are covered with micropillar arrays. SEM images of the fabricated sensor devices (i.e., pillar array 1 and pillar array 2) are depicted in Fig. $4 a$ and b. The dimensions of each micropillar on both sensor devices are $30 \mu \mathrm{m} \times 30 \mu \mathrm{m} \times 30 \mu \mathrm{m}$. Moreover, the pitch $p$ of pillar array 1 and pillar array 2 is $50 \mu \mathrm{m}$ and $150 \mu \mathrm{m}$, respectively. For both devices, a MEMS-based sensor was fabricated underneath a micropillar, as shown in the SEM images. The dimensions of the sensor are identical to those of the flat substrate depicted in Fig. $1 \mathrm{~b}$.

For the case of droplets with diameters of $2.2 \mathrm{~mm}$ and impacting on the sensor devices at a velocity of $0.56 \mathrm{~m} / \mathrm{s}$, the impact forces and the images captured using the highspeed camera are presented in Fig. 4c and d for the devices shown in Fig. 4a and b, respectively. The corresponding Supplementary videos S4 and S5 are provided in the Supplementary Information. Based on the images of the high-speed camera, it is evident that despite the identical diameters of the droplets and the identical impact velocities, bubble entrapment occurs in the case of pillar array 1 , but not in the case of pillar array 2. It can be observed that over the duration of the impact, the fractional resistance change of the cantilever of pillar array 2 was greater than that of the cantilever of pillar array 1 . This difference in the sensor signal amplitudes is caused because pillar array 2 is sparser than pillar array 1 ; thus, the average force acting on a micropillar of pillar array 2 is stronger than that acting on a micropillar of pillar array 1 . In the case of pillar array 1, vibration incorporated with bubble entrapment is observed in the sensor signal, as shown in the inset of the graph depicted in Fig. 4c. In contrast, in the case of pillar array 2, the sensor signal does not exhibit any detectable vibration. Most importantly, the results indicate the possibility of preventing bubble entrapment by alternating the surface morphology of the substrate; this is discussed in detail in the following section.

\section{Preventing bubble entrapment by surface morphology}

In this section, we discuss the possibility of using surface textures to control the occurrence of bubble 
$\mathbf{a}$

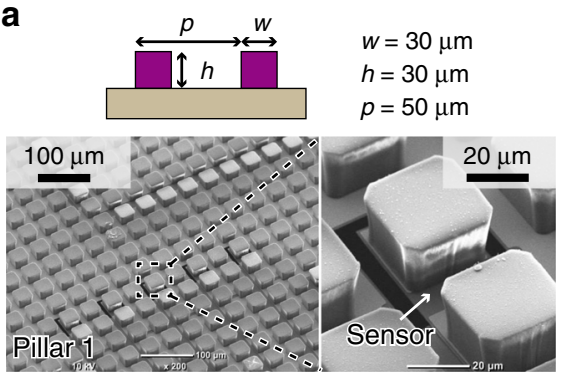

C
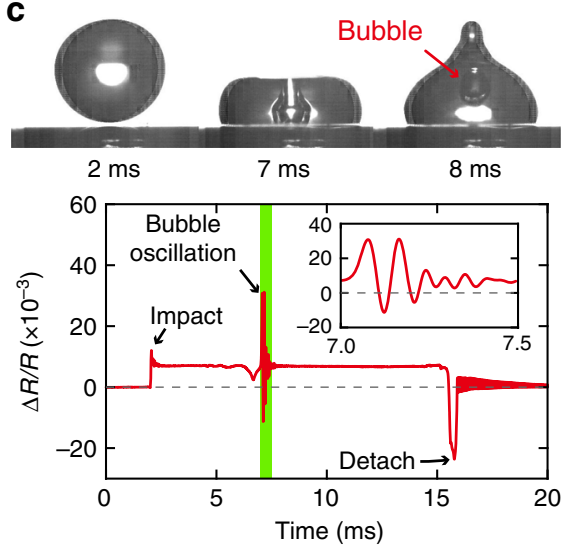

b

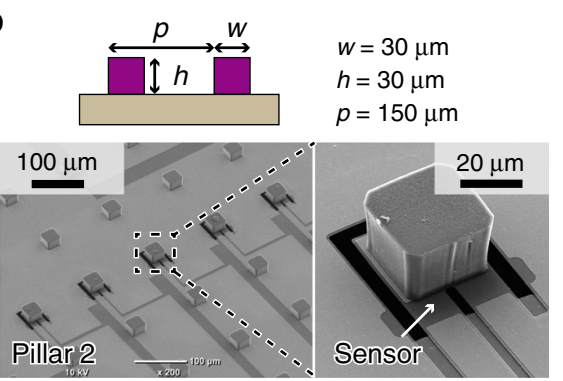

d
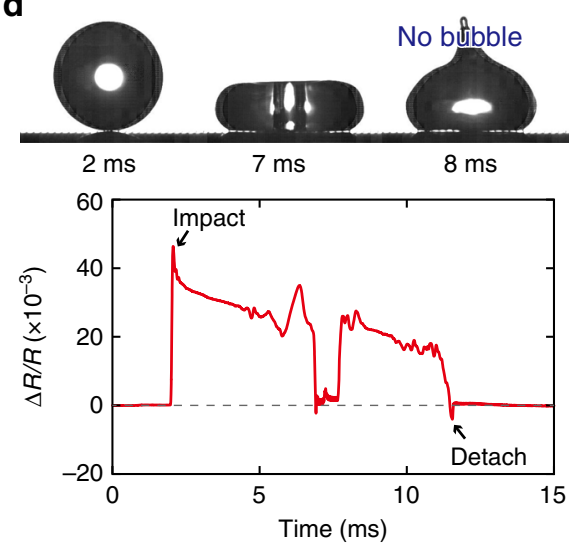

Fig. 4 Measurement results for sensors covered with micropillars. a, b SEM images of fabricated sensor devices with micropillar arrays on their surfaces. c, d Measurement results for droplets (diameter: $2.2 \mathrm{~mm}$ ) impacting the sensor devices at a velocity of $0.56 \mathrm{~m} / \mathrm{s}$

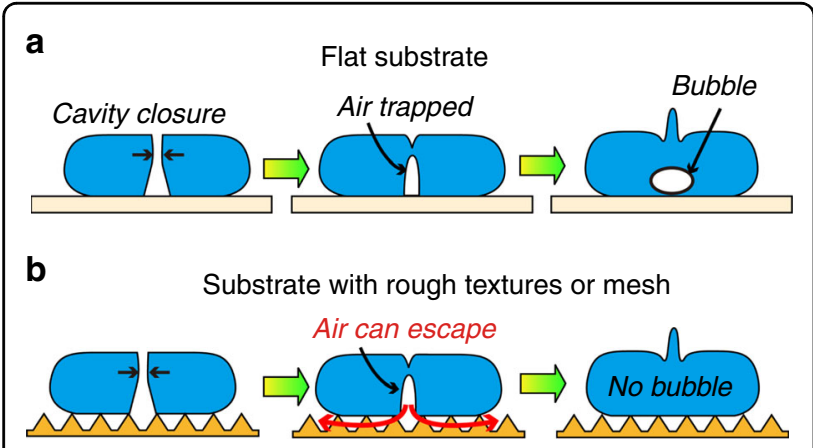

Fig. 5 Mechanism for prevention of bubble entrapment by controlling substrate morphology. Conceptual sketches of the air cavity closure and bubble entrapment during the recoil of a droplet impacting (a) a flat substrate and (b) a substrate with rough textures or through-holes

entrapment during the recoil of droplet impact. Because this type of bubble entrapment occurs when the air cavity formed after the spreading droplet is sealed as the liquid recoils to the center, the idea here is to create a pathway for the air inside the cavity to escape so that bubble entrapment can be prevented, as shown in Fig. 5. We tested this idea by investigating the impact of water droplets (diameter $D=2.3 \mathrm{~mm}$ ) on substrates with different surface morphologies: a flat $\mathrm{Si}$ wafer, $\mathrm{Si}$ wafers featuring micropillar arrays with different pitches and pillar heights, a mesh and an array of pyramid-shaped microstructures. The substrates used in the experiment were pillar array 1 (Fig. 4a), pillar array 2 (Fig. 4b), and the substrates illustrated in Fig. 6. In the experiment, the impact velocity $\mathrm{V}$ was systematically varied to change the Weber number within the range of 4-12, which covers the range where bubble entrapment during the recoil phase occurs for a substrate without textures.

The measurement results are shown in Fig. 7. Some snapshots from the high-speed camera showing the behaviors of the droplets impacting the five substrates illustrated in Fig. 6 at the same velocity $(0.56 \mathrm{~m} / \mathrm{s})$ are shown in Fig. 7a. The corresponding videos (Supplementary videos S6-S10) are provided in the Supplementary Information. The shape evolutions of these droplets exhibit little difference because all the substrates were superhydrophobic and the Weber numbers were the same. By the end of the spreading phase, the air cavities formed and became cylindrical shaped as the liquid recoiled. The difference in the droplet behaviors appeared after the air cavities collapsed: bubble entrapment occurred for the flat substrate ( $\mathrm{Si}$ wafer) and pillar array 3 , 

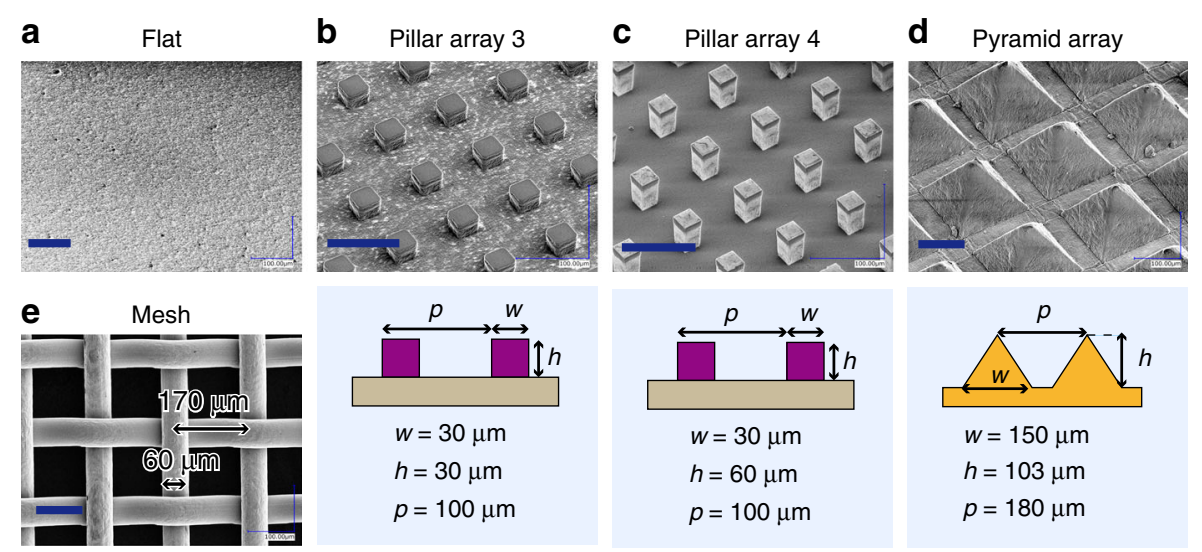

Fig. 6 Prepared substrates with different morphologies. SEM images of the substrates prepared for the experiment to investigate the effect of surface morphology on the occurrence of bubble entrapment. The scale bars are $100 \mu \mathrm{m}$

but not for pillar array 4, the pyramid-shaped microarray, or the mesh. It is not difficult to understand why bubble entrapment occurred for the $\mathrm{Si}$ wafer but not for the mesh; that is, the air inside the cavity could not escape when the Si wafer was used, but it could escape through the through-holes of the mesh. For the micropillar array and the pyramid-shaped microstructure array, the reason for the difference in the impact outcomes is the variation in the texture sizes and density. The results suggest that substrates with sufficiently large textures or large intervals between the textures can prevent bubble entrapment. For instance, as shown in Fig. 4, by increasing the interval of micropillars, it was possible to prevent bubble entrapment. Moreover, increasing the height of the pillar (e.g., pillar array 4 or the pyramid-shaped microstructure array compared with pillar array 3) also enables the prevention of bubble entrapment. The reason for this tendency is that the air inside the collapsing cavity can escape if the textures underneath the droplet are sufficiently large or sparse to maintain the air layer around the textures underneath the air cavity during its closure.

For example, Fig. 7b shows the close-up views of the droplets immediately before and after the moment of cavity closure for pillar array 3 and the pyramid-shaped microstructure array. It can be observed that in the case of pillar array 3 , immediately after the closure of the cavity at the top, the area on the contact area below the cavity was filled with liquid (black area in the snapshots), which prohibited the air from escaping. On the other hand, the air gaps surrounding the pyramid-shaped microstructures remained both before and after the closure of the air cavity, thus preventing bubble entrapment.

The effects of the Weber number on the occurrence of bubble entrapment for all substrates are shown in Fig. 8 . For a flat $\mathrm{Si}$ substrate and pillar array 1 (length: $30 \mu \mathrm{m}$, width: $30 \mu \mathrm{m}$, height: $30 \mu \mathrm{m}$, and pitch: $50 \mu \mathrm{m}$ ), bubble entrapment occurred for Weber numbers in the range of 7-11, whereas pillar array 3 (height: $30 \mu \mathrm{m}$ and pitch: $100 \mu \mathrm{m}$ ) was capable of preventing bubble entrapment for Weber numbers $<8$. In contrast, bubble entrapment did not occur when the droplet impacted pillar array 2 (height: $30 \mu \mathrm{m}$ and pitch: $150 \mu \mathrm{m}$ ), pillar array 4 (height: $60 \mu \mathrm{m}$ and pitch: $100 \mu \mathrm{m}$ ), the mesh and the pyramidshaped microstructure array over the entire range of the tested Weber numbers. This result indicates that one can prevent bubble entrapment during droplet recoil after impact using a substrate with sufficiently large or sparse textures or through-holes, which creates pathways for air to escape.

\section{Conclusion}

In this study, we experimentally examined the bubble entrapment induced by air cavity closure during the recoil phase of droplet impact. Using a piezoresistive cantilever fabricated on the substrate, we demonstrated that bubble entrapment is followed by an acoustic signal that can be detected by the cantilever. Therefore, using the cantilever, it is possible to detect bubble entrapment during droplet impact. Moreover, the size of the bubble can also be estimated from the frequency of the acoustic signal measured by the sensor. We also investigated the possibility of preventing bubble entrapment by creating pathways for the air in the closing cavity to escape to the underside of the contact area. The experimental results revealed that bubble entrapment could be prevented by using a substrate with through-holes, such as a mesh, or by decorating the substrate with sufficiently large or sparse textures. These findings not only improve our understanding of the physics of droplet impacts but are also useful in many applications, such as the prevention of bubble entrapment in inkjet printing and the utilization of bubbles in droplet mixing. 


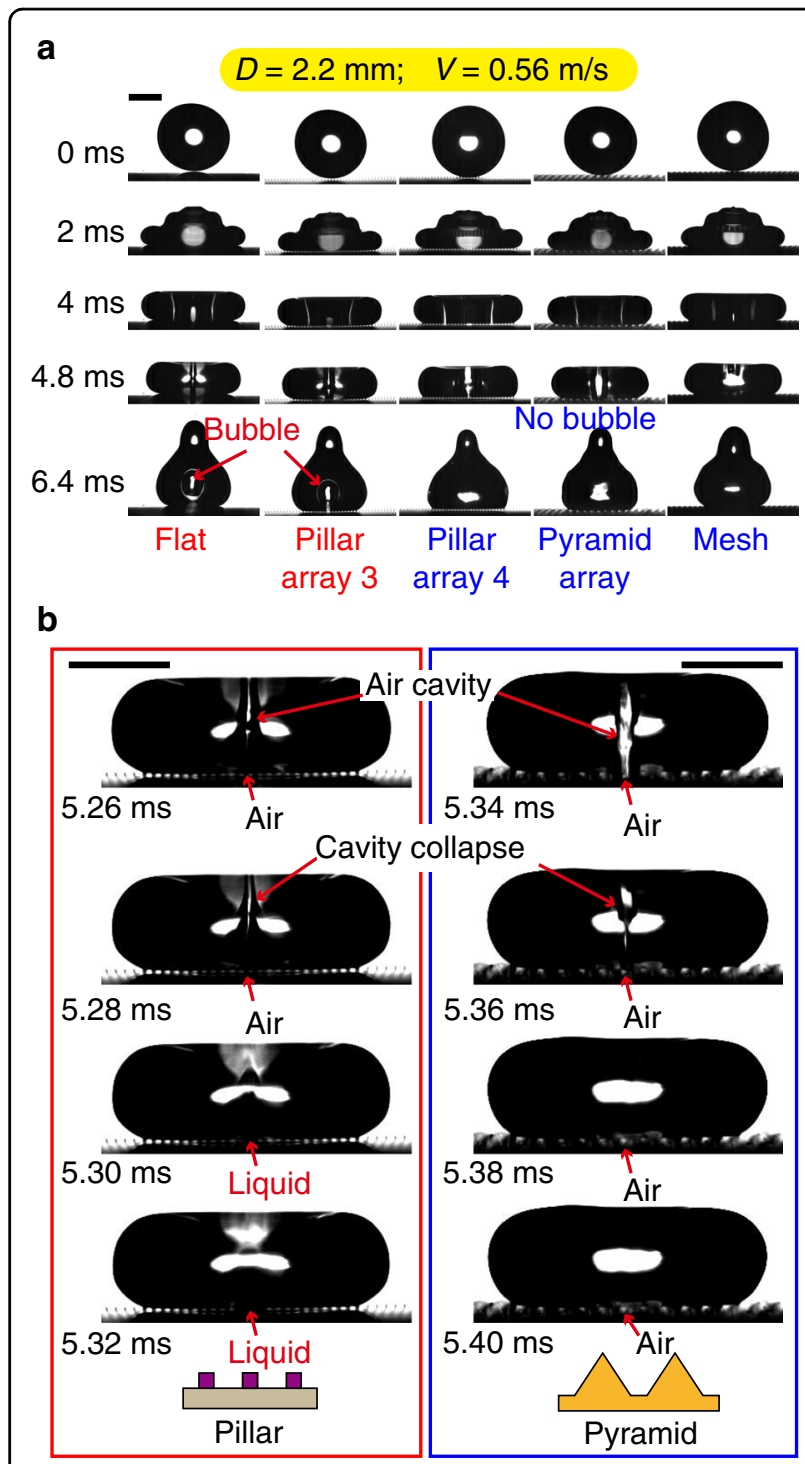

Fig. 7 Impacts of droplets on substrates with different morphologies. a Impacts of droplets with the same diameters on five substrates with different surface morphologies. The impact velocities were the same: $0.56 \mathrm{~m} / \mathrm{s}$. b Snapshots showing the cavity closure for the case of the pillar array 3 and a pyramid-shaped microstructure array. Scale bars: $1 \mathrm{~mm}$

\section{Materials and methods MEMS-based acoustic sensor}

The sensor used in this study was a MEMS-based piezoresistive cantilever, similar to the sensors that have been developed in our previous studies to measure forces generated by droplets and cells ${ }^{23-29}$. The cantilever was fabricated from a silicon-on-insulator wafer, whose device $\mathrm{Si}$, box, and handle Si layers' thicknesses were $300 \mathrm{~nm}$, $400 \mathrm{~nm}$, and $300 \mu \mathrm{m}$, respectively. The piezoresistive layer was formed by ion implantation, and metals $(\mathrm{Cr}$ and $\mathrm{Au})$ were deposited on the device $\mathrm{Si}$ layer using vacuum

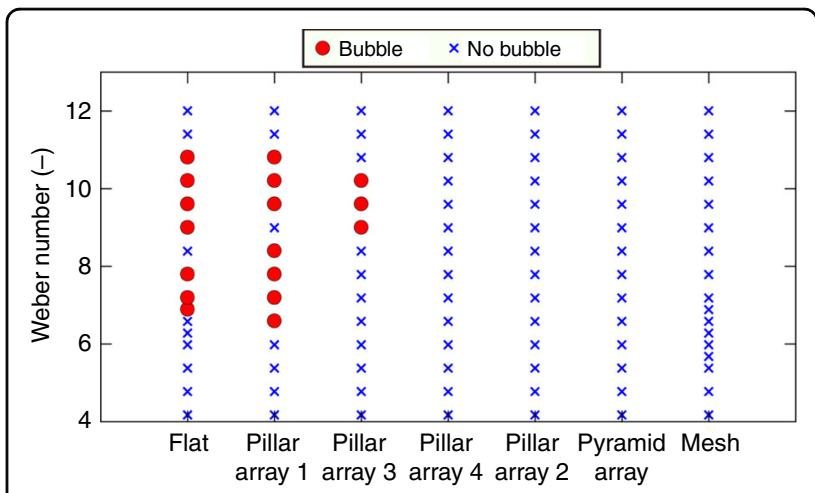

Fig. 8 Prevention of bubble entrapment by surface morphology. Effects of the Weber number and surface morphology on the occurrence of bubble entrapment

deposition. Next, the cantilever was formed by patterning the metal layers and etching the device Si layer. The piezoresistors were then formed by etching the metal layers at the root of the cantilever. For the fabrication of the sensors with micropillar arrays (Fig. 4a, b), a 30- $\mu \mathrm{m}$ thick SU-8 nega-photoresist layer was deposited and patterned on the sensor to form the micropillar arrays. Finally, the cantilever was released by etching the handle Si layer and box layer. The size of the cantilever was $35 \mu \mathrm{m} \times 30 \mu \mathrm{m}$, and the gap surrounding the cantilever was $\sim 1 \mu \mathrm{m}$. The size of the sensor chip was $10 \mathrm{~mm} \times$ $10 \mathrm{~mm}$, and the cantilever was located at the center of the chip. Before the experiment, the sensor chip was coated with hydrophobic nanoparticles to accomplish substrate superhydrophobicity ${ }^{23,29-31}$.

\section{Substrate preparation}

The micropillars, whose sizes were $30 \mu \mathrm{m} \times 30 \mu \mathrm{m} \times$ $30 \mu \mathrm{m}$, were fabricated by patterning an SU-8 nega-photoresist layer on the $\mathrm{Si}$ wafer. The pitch of the micropillar array was $100 \mu \mathrm{m}$. The array of pyramid-shaped microstructures was fabricated by casting Polydimethylsiloxane (PDMS) from an anisotropically etched $\mathrm{Si}$ wafer. The length of the pyramid's base, the height of each pyramid, and the interval between two adjacent pyramids were 150, $\sim 103$, and $180 \mu \mathrm{m}$, respectively. The mesh was purchased from Kyuho Corp. (Osaka, Japan). The diameter of the wire and the pitch of the mesh were $50 \mu \mathrm{m}$ and $170 \mu \mathrm{m}$, respectively. All substrates were coated with hydrophobic nanoparticles through a surface treatment process similar to that used for the sensor chip.

\section{Measurement setup}

The output of the sensor, corresponding to the fractional resistance change of the cantilever, was measured using a Wheatstone bridge circuit connected to an amplifier IC (Texas Instruments Inc., TX, USA, INA 217). 
The output of the measurement circuit was recorded at a sampling rate of 500,000 samples/s. In the experiment, water droplets were released from the tip of a syringe at different heights to change the impact velocity. The impact of the droplets was captured using a high-speed camera (Photron Ltd., Tokyo, Japan, FASTCAM SA-Z) at frame rates of 100,000 fps for measurements taken using the sensor and 50,000 fps for other measurements.

\section{Acknowledgements}

The photolithography masks were made using the University of Tokyo VLSI Design and Education Center (VDEC)'s 8 inch EB writer F5112 + VD01 donated by ADVANTEST Corporation. This work was supported by JSPS KAKENHI Grant Number $17 \mathrm{H} 04903$.

\section{Author contributions}

T.N. and M.I. conceived the idea. T.N. fabricated the sensors, designed and conducted the experiments, and analyzed the data; T.N. and M.I. wrote the paper.

\section{Conflict of interest}

The authors declare that they have no conflict of interest.

Supplementary information accompanies this paper at https://doi.org/ 10.1038/s41378-020-0158-y.

Received: 25 December 2019 Revised: 9 March 2020 Accepted: 30 March 2020

Published online: 29 June 2020

\section{References}

1. Yarin, A. L. DROP IMPACT DYNAMICS: splashing, spreading, receding, bouncing.... Annu. Rev. Fluid Mech. 38, 159-192 (2006).

2. Josserand, C. \& Thoroddsen, S. T. Drop impact on a solid surface. Annu. Rev. Fluid Mech. 48, 365-391 (2016).

3. Hendrix, M. H. W., Bouwhuis, W., van der Meer, D., Lohse, D. \& Snoeijer, J. H. Universal mechanism for air entrainment during liquid impact. J. Fluid Mech. 789, 708-725 (2016).

4. Hicks, P. D. \& Purvis, R. Air cushioning and bubble entrapment in threedimensional droplet impacts. J. Fluid Mech. 649, 135-163 (2010).

5. Lee, J. S., Weon, B. M., Je, J. H. \& Fezzaa, K. How does an air film evolve into a bubble during drop impact? Phys. Rev. Lett. 109, 204501 (2012).

6. Kolinski, J. M., Mahadevan, L. \& Rubinstein, S. M. Drops can bounce from perfectly hydrophilic surfaces. EPL (Europhys. Lett.) 108, 24001 (2014).

7. de Ruiter, J., Lagraauw, R., van den Ende, D. \& Mugele, F. Wettabilityindependent bouncing on flat surfaces mediated by thin air films. Nat. Phys 11, 48-53 (2015).

8. de Ruiter, J., Oh, J. M., van den Ende, D. \& Mugele, F. Dynamics of collapse of air films in drop impact. Phys. Rev. Lett. 108, 074505 (2012).

9. Klaseboer, E., Manica, R. \& Chan, D. Y. C. Universal behavior of the initial stage of drop impact. Phys. Rev. Lett. 113, 194501 (2014).

10. Bouwhuis, W. et al. Maximal air bubble entrainment at liquid-drop impact. Phys. Rev. Lett. 109, 264501 (2012).
11. Dam, D. B. V. \& Clerc, C. L. Experimental study of the impact of an ink-jet printed droplet on a solid substrate. Phys. Fluids 16, 3403-3414 (2004).

12. Mehdi-Nejad, V., Mostaghimi, J. \& Chandra, S. Air bubble entrapment under an impacting droplet. Phys. Fluids 15, 173-183 (2003).

13. RENARDY, Y. et al. Pyramidal and toroidal water drops after impact on a solid surface. J. Fluid Mech. 484, 69-83 (2003).

14. Hung, Y.-L., Wang, M.-J., Huang, J.-W. \& Lin, S.-Y. A study on the impact velocity and drop size for the occurrence of entrapped air bubbles-water on parafilm. Exp. Therm. Fluid Sci. 48, 102-109 (2013).

15. Chen, L., Li, L., Li, Z. \& Zhang, K. Submillimeter-sized bubble entrapment and a high-speed jet emission during droplet impact on solid surfaces. Langmuir 33, 7225-7230 (2017).

16. Chen, L., Bonaccurso, E., Deng, P. \& Zhang, H. Droplet impact on soft viscoelastic surfaces. Phys. Rev. E 94, 063117 (2016).

17. Chen, L. \& Li, Z. Bouncing droplets on nonsuperhydrophobic surfaces. Phys. Rev. E 82, 016308 (2010).

18. Bartolo, D., Josserand, C. \& Bonn, D. Singular jets and bubbles in drop impact. Phys. Rev. Lett. 96, 124501 (2006).

19. Won, T., Lee, K. Y. \& Chung, S. K. Acoustic bubble induced microstreaming for the enhancement of droplet mixing in Electrowetting (EW) microfluidic platforms. in 201920 th International Conference on Solid-State Sensors, Actuators and Microsystems \& Eurosensors XXXIII 65-67 (TRANSDUCERS \& EUROSENSORS XXXIII, 2019).

20. Minnaert, M. XVI. On musical air-bubbles and the sounds of running water. Lond., Edinb., Dublin Philos. Mag. J. Sci. 16, 235-248 (1933).

21. Agro, I. V., Theodore, F., Wilson, P. S. \& Palan, V. Measurement of the resonance frequency of single bubbles using a laser Doppler vibrometer. J. Acoustical Soc. Am. 123, EL121-EL125 (2008).

22. Spratt, K. S., Lee, K. M., Wilson, P. S. \& Wochner, M. S. On the resonance frequency of an ideal arbitrarily-shaped bubble. Proc. Meet. Acoust. 20, 045004 (2013).

23. Nguyen, T. \& Shimoyama, I. Reducing the contact time of droplet impact by active control of substrate motion. in 2018 The 31st IEEE International Conference on Micro Electro Mechanical Systems (MEMS) 202-205 (IEEE, 2018).

24. Matsudaira, $\mathrm{K}$. et al. MEMS piezoresistive cantilever for the direct measurement of cardiomyocyte contractile force. J. Micromech. Microeng. 27, 105005 (2017).

25. Tsukagoshi, T. et al. Cellular dynamics of bovine aortic smooth muscle cells measured using MEMS force sensors. J. Phys. D: Appl. Phys. 51, 145401 (2018).

26. Nguyen, T.-V., Nguyen, M.-D., Takahashi, H., Matsumoto, K. \& Shimoyama, I. Viscosity measurement based on the tapping-induced free vibration of sessile droplets using MEMS-based piezoresistive cantilevers. Lab a Chip 15, 3670-3676 (2015).

27. Nguyen, T.-V., Tsukagoshi, T., Takahashi, H., Matsumoto, K. \& Shimoyama, I. Depinning-induced capillary wave during the sliding of a droplet on a textured surface. Langmuir 32, 9523-9529 (2016).

28. Thanh-Vinh, N., Takahashi, H., Matsumoto, K. \& Shimoyama, I. Two-axis MEMSbased force sensor for measuring the interaction forces during the sliding of a droplet on a micropillar array. Sens. Actuators a-Phys. 231, 35-43 (2015).

29. Mouterde, T. et al. How merging droplets jump off a superhydrophobic surface: measurements and model. Phys. Rev. Fluids 2, 112001 (2017).

30. Nguyen, T. V., Mouterde, T., Takahashi, H., Quéré, D. \& Shimoyama, I. Jumping force of coalescing droplets on a superhydrophobic surface. in 2017 IEEE 30th International Conference on Micro Electro Mechanical Systems (MEMS) 95-98 (IEEE, 2017).

31. Nguyen, T. \& Shimoyama, I. Maximum pressure caused by droplet impact is dependent on the droplet size. in 2019 20th International Conference on SolidState Sensors, Actuators and Microsystems \& Eurosensors XXXIII, 813-816 (TRANSDUCERS \& EUROSENSORS XXXIII, 2019). 